
for Mechanical Engineering

\title{
Design and Analysis of a Tendon-driven Snake-arm Robot Based on Spherical Magnet
}

\begin{tabular}{|c|c|}
\hline Journal: & Transactions of the Canadian Society for Mechanical Engineering \\
\hline Manuscript ID & TCSME-2020-0084.R1 \\
\hline Manuscript Type: & Article \\
\hline $\begin{array}{l}\text { Date Submitted by the } \\
\text { Author: }\end{array}$ & 27-Mar-2021 \\
\hline Complete List of Authors: & $\begin{array}{l}\text { Liu, Bei; Changsha University of Science and Technology, School of } \\
\text { Automotive and Mechanical Engineering } \\
\text { Yin, Lairong; Changsha University of Science and Technology, School of } \\
\text { Automotive and Mechanical Engineering } \\
\text { Huang, Long; Changsha University of Science and Technology, School of } \\
\text { Automotive and Mechanical Engineering } \\
\text { Zhang, Peng; Changsha University of Science and Technology, School of } \\
\text { Automotive and Mechanical Engineering } \\
\text { Yi, Kefu; Changsha University of Science and Technology, School of } \\
\text { Automotive and Mechanical Engineering; Changsha University of Science } \\
\text { and Technology, Hunan Provincial Key Laboratory of Intelligent } \\
\text { Manufacturing Technology for High-performance Mechanical Equipment }\end{array}$ \\
\hline Keywords: & $\begin{array}{l}\text { spherical magnets, tendon-driven approach, passive compliance, snake- } \\
\text { arm robot }\end{array}$ \\
\hline $\begin{array}{r}\text { Is the invited manuscript for } \\
\text { consideration in a Special } \\
\text { Issue? : }\end{array}$ & Not applicable (regular submission) \\
\hline
\end{tabular}

\section{SCHOLARONE ${ }^{\text {IM }}$ Manuscripts}




\section{Design and Analysis of a Tendon-driven Snake-arm Robot Based on Spherical Magnet}

Bei Liu ${ }^{1}$, Lairong Yin ${ }^{1}$, Long Huang ${ }^{1 *}$, Peng Zhang $^{1}$, KefuYi $^{1,2}$

${ }^{1}$ School of Automotive and Mechanical Engineering, Changsha University of Science and Technology, Changsha,

$$
\text { 410114, China }
$$

${ }^{2}$ Hunan Provincial Key Laboratory of Intelligent Manufacturing Technology for High-performance Mechanical Equipment, Changsha University of Science and Technology, Changsha 410114, China Corresponding author: Long Huang (E-mail: huanglongin@foxmail.com) 


\begin{abstract}
The tendon-driven snake-arm robot can achieve multiple degrees of freedom (DOF) bending motion with a compact structure, which enables the robot to be widely applied in confined environments. However, if a conventional tendon-driven snake-arm robot is subject to a lateral force on the distal end, it will experience passive compliance. In this paper, a 2-DOF rolling joint is proposed based on the opposite-pole attraction of spherical magnets, which has a relatively simple structure than traditional joints. By serial connecting the 2-DOF rolling joints, a novel snake-arm robot is designed utilizing a tendon-driven approach. The kinematic model and workspace of the snake-arm robot are obtained, and the bending motion is validated. Based on the kinematic model, it is theoretically proved that the proposed robot can avoid passive compliance. In addition, this feature is verified through load experiments on the developed prototype.
\end{abstract} Keywords: spherical magnets, tendon-driven approach, passive compliance, snake-arm robot 


\section{INTRODUCTION}

The tendon-driven snake-arm robot has been widely applied in the fields of medical surgery (Camarillo et al., 2008, Huang et al., 2021) and industrial applications (Simaan, 2005) due to its compact structure and high flexibility. Usually, the tendon-driven snake-arm robot can realize multi-DOF bending with serial connecting of revolute joints or flexible backbones (Yoon and Yi. 2009, Fei et al., 2018; Lei and Du, 2011; Kato et al., 2014; Nguyen and Burgner-Kahrs 2015), and the later structure is also referred to as continuum robots. Based on compliant joints, Dong et al. proposed a class of snake-arm robots and analyzed their stiffness (Dong et al., 2014, 2016). Li et al. developed a new tendon-driven continuum robot with spherical joints and a flexible backbone in 2013 (Li et al., 2013). Yamashita et al. designed a forceps manipulator using a wire-restraint mechanism with a pair of guide-wire (Yamashita et al., 2006). However, most of the prior prototypes adopted 1-DOF or 2-DOF revolute joints or flexible backbones to construct the tendon-driven robot, which usually have the potential problem of passive compliance (Kim et al., 2014). When the distal end of the robot is subject to a lateral force, even if the inputs of the robot remain the same, the configuration may deviate from its original state, which causes the uncertainty of the operation. In other words, the tendon tension in the conventional snake-arm robots could not resist the lateral force. Therefore, it is important to avoid passive compliance during the structural design processes.

To address this issue, Kim et al. reported a variable neutral line method to avoid passive compliance; utilizing this method, a tendon-driven continuum robot is designed by connecting identical cylindrical rolling joints in series (Kim et al., 2014). However, the robot needs considerable tendon tensions to keep pure rolling motion within the adjacent rolling joints. Suh et al. developed a cylindrical rolling joint using elastic fixtures (Suh et al., 2015), which can generate a smooth rolling motion without passive compliance. However, it is relatively complex to determine and assemble the elastic fixture. In this paper, we propose a design of a 2-DOF rolling joint with spherical magnets. Based on the design, a novel tendon-driven snake-arm robot is presented, which can avoid passive compliance yet has a compact structure and large workspace. Owing to the mutual attraction of adjacent magnets, the snake-arm robot can maintain the pure rolling motion in the joints without large tendon tension, which is beneficial to the lifespan of the tendons.

The remaining part of this paper is organized as follows. Section 2 briefly introduces passive compliance in the conventional tendon-driven snake-arm robot. Section 3 explains the structural design of the snake-arm robot, which is inspired by the motion of adjacent attracting spherical magnets. Section 4 conducts the kinematics of the snake-arm robot. Section 5 theoretically proves that the proposed robot can avoid passive compliance. Section 6 validates the structure through load experiments.

\section{PASSIVE COMPLIANCE OF TENDON-DRIVEN ROBOTS}

A 1-DOF conceptual design of a tendon-driven snake-arm robot is shown in Fig.1, including several identical revolute joints and springs fixed around them (Walker, 2013). To explain the passive compliance and tendon slack in the traditional tendon-driven snake-arm robot, considering the robot shown in Fig.1 as an example, we set the released 
and tightened amount of each tendon as the input of the robot, and the robotic configuration as the output. In the straight configuration, the lengths of the tendons on both sides are equal $\left(L_{l}=L_{r}\right)$ (Fig. 1(a)). When the non-loaded robot bends to the left, as shown in Fig.1(b), the left tendon tightens and the right tendon releases, which means that the tendon length changes of both sides are negative and positive respectively $\left(\Delta L_{l}<0\right.$ and $\left.\Delta L_{r}>0\right)$. Accordingly, in a single joint, the tendon length changes of both sides are negative and positive respectively $\left(\Delta l_{l}<0\right.$ and $\left.\Delta l_{r}>0\right)$. Based on the joint geometry, it can be obtained that the tightened amount of the left tendon is less than the released amount of the right tendon $\left(\Delta l_{l}+\Delta l_{r}<0\right)$ for any bending configuration of this robot. Therefore, the sum of the tendon length changes of both sides in the robot is negative $\left(\Delta L_{l}+\Delta L_{r}<0\right)$. If this snake-arm robot in the straight configuration is subject to lateral forces on the distal end, it will change to the S-configuration shown in Fig. 1(c), which can be considered as the serial combination of a left-bending section and a right-bending section. During the transition of configurations, if the input of the robot remains the same, both tendons will become slack $\left(L_{l 1}{ }^{\prime \prime}+L_{l 2}{ }^{\prime \prime}=L_{r 1}{ }^{\prime \prime}+L_{r 2}{ }^{\prime \prime}<2 L_{l}=2 L_{r}\right)$. This is the passive compliance of the snake-arm robot, which also exists in snake-arm robots with flexible backbones. If $\Delta L_{l}+\Delta L_{r}$ is positive for a specific snake-arm robot, it will not change from the straight configuration to S-configuration under lateral forces.

\section{ROBOT DESIGN}

If two spherical magnets contact at their opposite poles (Fig. 2(a)), the magnetic attraction will make this state to be a stable state. If a certain lateral force $F$ is applied in any direction on the spherical magnet, the magnet will undergo a pure rolling motion on the spherical surface, as shown in Fig. 2(b). Owing to the magnetic field, the spherical magnets will automatically return to the initial stable state when the lateral force $F$ is removed.

Inspired by the motion characteristic of the spherical magnets, a novel snake-arm robot is developed, as shown in Fig. 3. The snake-arm robot consists of a 2-DOF manipulator and a driving module. The manipulator is composed of several identical rolling joints which are connected in series by opposite poles in adjacent spherical magnets. One rolling joint contains two modules with two spherical magnets and four disks. Each disk in Fig. 3(b) has a hole matching the spherical magnets and three small holes for the tendons (tendon A, tendon B, and tendon C). The spherical magnet and disks are fixed to each other. In the straight configuration in Fig. 3(a) and Fig. 3(c), the central axis of the disks coincides with the $N$ and $S$ poles of the spherical magnet. The CAD model of the tendon-driven snake-arm robot is shown in Fig.3(a). Each tendon goes through the tendon holes of each disk, and it is fixed to the distal end of the manipulator. In the initial state, a certain pre-tensioning force is applied to ensure that there is no slack in three tendons. The attraction between spherical magnets can reduce the tendon tension in conventional revolute joints, which can increase the lifespan of the tendon.

\section{KINEMATICS}

Unlike the traditional serial robots, the kinematic model of the snake-arm robot needs to establish the mapping between the actuator space (i.e. the robotic inputs $\left(Z_{A}, Z_{B}, Z_{C}\right)$ ), configuration space (i.e. bending angle $\theta$ and bending 
direction angle $\varphi$ ), and task space (i.e. the distal end position $(x, y, z)$ ), as shown in Fig. 4 . In this section, the kinematics of the robot is presented based on the modeling of the rolling joint.

\subsection{Kinematics of the Rolling Joint}

Herein the radius of the spherical magnet, diameter and the thickness of the disk are denoted by $r, D$ and $a$, respectively. Fig. 5 shows the initial state of the rolling joint which comprises of the $i^{\text {th }}$ module and the $(i+1)^{\text {th }}$ module. Two D-H coordinate systems, $\{i\}$ and $\{i+1\}$, are affiliated to the modules. The origins of the coordinate systems coincide with the centers of spherical magnets; the $x$-axes pass through the hole center of tendon A and the $z$-axes point at the $N$ pole. In the initial state, the lengths of the tendons in the rolling joint are equal to the diameter of the magnet.

$l_{A 0}=l_{B 0}=l_{C 0}=2 r$

where $L_{A 0}, L_{B 0}$, and $L_{A 0}$ represent the straight tendon lengths in the rolling joint.

When the rolling joint changes to a bending configuration in an arbitrary direction, the projection of vector $\boldsymbol{O}_{i} \boldsymbol{O}_{i+1}$ on $x_{i} y_{i}$-plane is defined as the bending vector $\boldsymbol{w}$. Furthermore, the plane formed by $\boldsymbol{O}_{i} \boldsymbol{O}_{i+1}$ and vector $\boldsymbol{w}$ is defined as the bending plane, as shown in Fig. 6 . The bending direction angle from $x_{i}$-axis to vector $\boldsymbol{w}$ is denoted by $\varphi$, and the bending angle in the single rolling joint is denoted by $\theta$. The diameter of the distribution circle of the tendons is denoted by $d$.

As shown in Fig. 6(a), the scalar projections of the vectors $\boldsymbol{F} \boldsymbol{A}, \boldsymbol{F B}$ and $\boldsymbol{F C}$ in the direction of bending vector $\boldsymbol{w}$ are as follows.

$$
\left\{\begin{array}{l}
d_{F G}=\frac{d}{2} \cos \varphi \\
d_{F E}=\frac{d}{2} \cos \left(\varphi+\frac{2 \pi}{3}\right) \\
d_{F D}=\frac{d}{2} \cos \left(\varphi+\frac{4 \pi}{3}\right)
\end{array}\right.
$$

According to the geometric structure of the rolling joint on the bending plane, the quadrilateral $M F O_{l} \mathrm{O}_{2}, M F D Q$, $M F G H$ and $M F E K$ are all isosceles trapezoids. Thence, the lengths of the neutral line $M F$ are obtained as

$$
|M F|=2\left(r-a \cos \frac{\theta}{2}\right)
$$

Therefore, the lengths of the tendons can be expressed by $\theta$ and $\varphi$ as follows:

$$
\left\{\begin{array}{l}
l_{A}=2\left(r+a-a \cos \frac{\theta}{2}\right)-d \cos \varphi \sin \frac{\theta}{2} \\
l_{B}=2\left(r+a-a \cos \frac{\theta}{2}\right)-d \cos \left(\varphi+\frac{2 \pi}{3}\right) \sin \frac{\theta}{2} \\
l_{C}=2\left(r+a-a \cos \frac{\theta}{2}\right)-d \cos \left(\varphi+\frac{4 \pi}{3}\right) \sin \frac{\theta}{2}
\end{array}\right.
$$

where $l_{A}, l_{B}$, and $l_{C}$ represent the lengths of three tendons in the rolling joint, respectively; the range of the angle $\varphi$ is from 0 to $2 \pi$.

When the lower edge of the upper disk collides with the upper edge of the lower disk, the single rolling joint reaches the maximum bending angle, as shown in Fig. 7. 
Therefore, the maximum bending angle of the single rolling joint is calculated as

$$
\theta_{\max }=2\left(\arctan \frac{r}{\sqrt{a^{2}+\left(\frac{D}{2}\right)^{2}-r^{2}}}-\arcsin \frac{a}{\sqrt{a^{2}+\left(\frac{D}{2}\right)^{2}}}\right)
$$

\subsection{Forward Kinematics}

In the forward kinematics of the snake-arm robot, it is necessary to map from the actuator space to the configuration space and then from the configuration space to the task space. Assuming that the inputs of the robot are $Z_{A}, Z_{B}$, and $Z_{C}$, the total lengths of each tendon in the snake-arm robot is $V$, which is a constant.

$$
Z_{A}+L_{A}=Z_{B}+L_{B}=Z_{C}+L_{C}=V
$$

where $L_{A}, L_{B}$, and $L_{C}$ represent the lengths of three tendons in the manipulator.

According to Eq. (4), the length of each tendon in the manipulator can be expressed as

$$
\left\{\begin{array}{l}
L_{A}=\sum_{i=1}^{n}\left(2\left(r+a-a \cos \frac{\theta}{2}\right)-d \sin \frac{\theta}{2} \cos \varphi\right) \\
L_{B}=\sum_{i=1}^{n}\left(2\left(r+a-a \cos \frac{\theta}{2}\right)-d \sin \frac{\theta}{2} \cos \left(\varphi+\frac{2 \pi}{3}\right)\right) \\
L_{C}=\sum_{i=1}^{n}\left(2\left(r+a-a \cos \frac{\theta}{2}\right)-d \sin \frac{\theta}{2} \cos \left(\varphi+\frac{4 \pi}{3}\right)\right)
\end{array}\right.
$$

where $L_{A}, L_{B}$, and $L_{C}$ represent the lengths of the three tendons using the $\theta$ and $\varphi$.

Hence, the angles $\theta$ and $\varphi$ can be calculated as

$$
\left\{\begin{array}{l}
\theta=2 \arccos \left(1+\frac{r}{a}-\frac{L_{A}+L_{B}+L_{C}}{6 n a}\right) \\
\varphi=\arccos \frac{L_{B}+L_{\mathrm{C}}-2 L_{A}}{3 n d \sqrt{1-\left(1+\frac{r}{a}-\frac{L_{A}+L_{B}+L_{\mathrm{C}}}{6 n a}\right)^{2}}}
\end{array}\right.
$$

Herein the D-H coordinate systems $\{0\} \sim\{n\}$ are attached to each module, as shown in Fig. $8 .\{0\}$ denotes the base coordinate system, and $\{n\}$ denotes the distal end coordinate system.

According to the geometric structure of the manipulator, the position of $O_{n}$ can be expressed as

$$
{ }_{n}^{0} \boldsymbol{p}=\left[\begin{array}{c}
p_{x} \\
p_{y} \\
p_{z}
\end{array}\right]=\left[\begin{array}{c}
2 r \cos \varphi \sum_{i=1}^{n} \sin \frac{2 i-1}{2} \theta \\
2 r \sin \varphi \sum_{i=1}^{n} \sin \frac{2 i-1}{2} \theta \\
2 r \sum_{i=1}^{n} \cos \frac{2 i-1}{2} \theta
\end{array}\right]
$$

Since the manipulator consists of several identical rolling joints in series, the bending angle of each rolling joint is equal. This is based on the assumption that the tendon tension acting on each rolling module is equal. When the manipulator is bending in any direction, the bending motion of all the rolling joints is on the same bending plane. Then 
the rotation matrix from the base coordinate $O_{0}$ to the distal end coordinate $O_{n}$ can be obtained as

$$
{ }_{n}^{0} \boldsymbol{R}=\left[\begin{array}{ccc}
\cos \frac{(2 n-1) \theta}{2} \cos \varphi & -\sin \varphi \cos \frac{(2 n-1) \theta}{2} & \sin \frac{(2 n-1) \theta}{2} \\
\sin \varphi & \cos \varphi & 0 \\
-\sin \frac{(2 n-1) \theta}{2} \cos \varphi & \sin \frac{(2 n-1) \theta}{2} \sin \varphi & \cos \frac{(2 n-1) \theta}{2}
\end{array}\right]
$$

\subsection{Inverse Kinematics}

The inverse kinematics is to establish the mapping from task space to configuration space and the mapping from configuration space to actuator space. According to the previous section, the configuration space can be easily mapped from the task space.

Note that

$$
\left\{\begin{array}{l}
\sum_{i=1}^{n} \cos \frac{i \theta}{2}=\frac{\cos \left(\frac{n+1}{4} \theta\right) \sin \left(\frac{n}{4} \theta\right)}{\sin \frac{\theta}{4}} \\
\sum_{i=1}^{n} \sin \frac{i \theta}{2}=\frac{\sin \left(\frac{n+1}{4} \theta\right) \sin \left(\frac{n}{4} \theta\right)}{\sin \frac{\theta}{4}}
\end{array}\right.
$$

Hence, by substituting Eq. (11) into Eq. (9), the angles $\theta$ and $\varphi$ can be solved inversely as follows:

$$
\left\{\begin{array}{l}
\theta=\frac{4}{n+1} \arctan \frac{\sqrt{x^{2}+y^{2}}}{z} \\
\varphi=\arctan \frac{y}{x}
\end{array}\right.
$$

According to Eq. (7), the lengths of each tendon in the manipulator can be obtained, and the actuator space can be finally obtained by Eq. (6).

\subsection{Workspace}

The workspace of the snake-arm robot is closely related to the number of rolling joints, joint structure, and bending angle of each joint. Based on the kinematics and the structure, the workspace with preferred the bending angle of each joint $\left(\theta \in\left[0,20^{\circ}\right]\right)$ is simulated by MATLAB, as shown in Fig. 9 . The workspace of the manipulator is a large spherical surface. Fig. 9 (b) shows the different bending configuration of the manipulator on the $x-z$ plane with a bending angle $\theta$ of $\pm 5^{\circ}, \pm 10^{\circ}, \pm 15^{\circ}$, and $\pm 20^{\circ}$, which explains the bending configurations between $-\theta$ and $\theta$ are symmetric. As shown in Fig. 9, the manipulator can achieve a 2-DOF bending motion which is also validated by the experience with different configurations $\left(\theta=15^{\circ}\right)$.

\section{PASSIVE COMPLIANCE ANALYSIS OF THE ROBOT}

If the tendon-driven snake-arm robot is subject to a lateral force on its distal end while its inputs remain the same, the robot should be able to keep its current configuration. For the proposed robot in the arbitrary configuration, we assume that it changes to an unknown configuration under a certain lateral force. The bending angle changes of the rolling joints are denoted by $\left\{\Delta \theta_{1}, \Delta \theta_{2}, \Delta \theta_{3}, \ldots, \Delta \theta_{n}\right\}$. The bending direction angle changes of the rolling joints are 
denoted by $\left\{\Delta \varphi_{1}, \Delta \varphi_{2}, \Delta \varphi_{3}, \ldots, \Delta \varphi_{n}\right\}$. Based on the length change analysis, the following derivation will prove that when $\left\{\Delta \theta_{1}, \Delta \theta_{2}, \Delta \theta_{3}, \ldots, \Delta \theta_{n}\right\}$ only is zero, the proposed snake-arm robot can avoid passive compliance. According to Eq. (7), the lengths of the three tendons can be rewritten as

$$
\left\{\begin{array}{l}
L_{A 1}=\sum_{i=1}^{n}\left(2\left(r+a-a \cos \frac{\theta+\Delta \theta_{i}}{2}\right)-d \sin \frac{\theta+\Delta \theta_{i}}{2} \cos \left(\varphi+\Delta \varphi_{i}\right)\right) \\
L_{B 1}=\sum_{i=1}^{n}\left(2\left(r+a-a \cos \frac{\theta+\Delta \theta_{i}}{2}\right)-d \sin \frac{\theta+\Delta \theta_{i}}{2} \cos \left(\varphi+\Delta \varphi_{i}+\frac{2 \pi}{3}\right)\right) \\
L_{C 1}=\sum_{i=1}^{n}\left(2\left(r+a-a \cos \frac{\theta+\Delta \theta_{i}}{2}\right)-d \sin \frac{\theta+\Delta \theta_{i}}{2} \cos \left(\varphi+\Delta \varphi_{i}+\frac{4 \pi}{3}\right)\right)
\end{array}\right.
$$

Hence, the length changes of each tendon after the application of the lateral force are denoted as

$$
\left\{\begin{array}{l}
\Delta L_{A}=L_{A 1}-L_{A}=2 a \sum_{i=1}^{n}\left(\cos \frac{\theta}{2}-\cos \frac{\theta+\Delta \theta_{i}}{2}\right)-d \sum_{i=1}^{n}\left(\sin \frac{\theta+\Delta \theta_{i}}{2}\right) \cos \left(\varphi+\Delta \varphi_{i}\right)+d \cos (\varphi) \sum_{i=1}^{n}\left(\sin \frac{\theta}{2}\right) \\
\Delta L_{B}=L_{B 1}-L_{B}=2 a \sum_{i=1}^{n}\left(\cos \frac{\theta}{2}-\cos \frac{\theta+\Delta \theta_{i}}{2}\right)-d \sum_{i=1}^{n}\left(\sin \frac{\theta+\Delta \theta_{i}}{2}\right) \cos \left(\varphi+\Delta \varphi_{i}+\frac{2 \pi}{3}\right)+d \cos \left(\varphi+\frac{2 \pi}{3}\right) \sum_{i=1}^{n}\left(\sin \frac{\theta}{2}\right) \\
\Delta L_{C}=L_{C 1}-L_{C}=2 a \sum_{i=1}^{n}\left(\cos \frac{\theta}{2}-\cos \frac{\theta+\Delta \theta_{i}}{2}\right)-d \sum_{i=1}^{n}\left(\sin \frac{\theta+\Delta \theta_{i}}{2}\right) \cos \left(\varphi+\Delta \varphi_{i}+\frac{4 \pi}{3}\right)+d \cos \left(\varphi+\frac{4 \pi}{3}\right) \sum_{i=1}^{n}\left(\sin \frac{\theta}{2}\right)
\end{array}\right.
$$

The sum of $\Delta L_{A}, \Delta L_{B}$, and $\Delta L_{C}$ is obtained as

$$
\Delta L_{A}+\Delta L_{B}+\Delta L_{C}=6 a \sum_{i=1}^{n}\left(\cos \frac{\theta}{2}-\cos \frac{\theta+\Delta \theta_{i}}{2}\right)=6 a \sum_{i=1}^{n}\left(\cos \frac{\theta}{2}\left(1-\cos \frac{\Delta \theta_{i}}{2}\right)+\sin \frac{\theta}{2} \sin \frac{\Delta \theta_{i}}{2}\right)
$$

Since the range of $\theta / 2$ is $\left[0,45^{\circ}\right), \cos (\theta / 2)$ is larger than $\sin (\theta / 2)$. According to the Eq. (14), when the unloaded configuration of the manipulator is an arbitrary bending configuration $(\theta \neq 0)$, at least one of $\left\{\Delta L_{A}, \Delta L_{B}, \Delta L_{C}\right\}$ are positive. This means that at least one of $\left\{L_{A}, L_{B}, L_{C}\right\}$ will increase during the deformation, which is impossible when the inputs of the robot remain the same.

If the unloaded configuration is the straight configuration $(\theta=0)$, and at least one of $\left\{\Delta \theta_{1}, \Delta \theta_{2}, \Delta \theta_{3}, \ldots, \Delta \theta_{n}\right\}$ are nonzero, then Eq. (15) can be rewritten as

$$
\Delta L_{A}+\Delta L_{B}+\Delta L_{C}=6 a \sum_{i=1}^{n}\left(1-\cos \frac{\Delta \theta_{i}}{2}\right)
$$

Since $a>0$, it is also proved that at least one of $\left\{L_{A}, L_{B}, L_{C}\right\}$ will increase during the deformation, which is also impossible when the inputs of the robot remain the same. Therefore, $\left\{\Delta \theta_{1}, \Delta \theta_{2}, \Delta \theta_{3}, \ldots, \Delta \theta_{n}\right\}$ must be equal to zero, and the robot is able to keep the current configuration.

As shown in Fig. 10, if the proposed robot in the straight configuration changes to the $S$ configuration under a lateral force, $\left\{L_{A}, L_{B}, L_{C}\right\}$ will increase during the deformation, and the inputs of the robot need to be adjusted. Therefore, it is proved that the proposed robot is free of passive compliance.

\section{VERIFICATION AND DISCUSSION}

To validate the proposed structure, a prototype of the snake-arm robot is established. As shown in Fig. 11, the prototype is composed of a driving module and a 2-DOF manipulator. The manipulator consists of 6 rolling joints connected in series 
by a stainless steel tendon with $1 \mathrm{~mm}$ in diameter. The total length of the manipulator is $180 \mathrm{~mm}$. Each joint contains two spherical magnets (ferrite material) with $25 \mathrm{~mm}$ in diameter. The spherical magnets are fixed to the aluminum disks with $36 \mathrm{~mm}$ in diameter and $5 \mathrm{~mm}$ in thickness. The diameter of the tendon distribution circle on the disk is $31 \mathrm{~mm}$.

To study the effects of the lateral forces, load experiments are performed on the prototype in linear and bending configurations. In the experiments, three gear motors (rated speed: $10 \mathrm{r} / \mathrm{min}$, torque: $70 \mathrm{Kg} \cdot \mathrm{cm}$ ) are used to drive the snakearm robot. As shown in Fig. 12, in the linear configuration, $2 \mathrm{~N}, 4 \mathrm{~N}$, and $5 \mathrm{~N}$ weights are applied on the distal end of the prototype respectively. It is obvious that the prototype does not conform to the $S$-shaped configuration. In comparison with the unloaded straight configuration shown in Fig. 12 (a), there is a small displacement (less than $1 \mathrm{~mm}$ ) of the distal end in the load direction. The reason is that the hole diameter in the disks is relatively larger than the diameter of the tendons which enables the two parts to slide when assembled. It is noteworthy that the precision of the prototype can be improved by adopting high-precision manufacture, assembly, and tensioning techniques.

In addition, the load experiments of the robot in different bending configurations are performed. Four bending configurations are selected, as shown in Fig. 13(a), Fig. 13(c), Fig. 13(e), and Fig. 13(g). When a 5 N vertical force acts on the distal end of the prototype, the configurations are shown in Fig. 13(b), Fig. 13(d), Fig. 13(f), and Fig. 13(h). The loaded configurations are much the same with the unloaded configurations, which proves that the prototype is free of passive compliance.

\section{CONCLUSION}

Inspired by the motion of two contacting spherical magnets, this paper presents a novel rolling joint structure, which can be connected in series to form a tendon-driven snake-arm robot. The maximum bending angle and the workspace of the snake-arm robot are obtained based on the kinematics. Based on the kinematic model, it is proved that the tendon-driven snake-arm robot is able to avoid passive compliance. The load experiments are performed on the straight and bending configurations of the developed prototype. The experimental results validate the feasibility of the proposed design.

\section{ACKNOWLEDGES}

This work is supported by the National Natural Science Foundation of China (No. 51805047 and 51705034 ), the Natural Science Foundation of Hunan province (No. 2019JJ50664, 2018JJ3548, 2018JJ3553), the Open Research Project of the State Key Laboratory of Industrial Control Technology, Zhejiang University, China (ICT2021B02).

\section{REFERENCES}

D. B. Camarillo, C. F. Milne, C. R. Carlson, et al. 2008. Mechanics modeling of tendon-driven continuum manipulators. IEEE Transactions on Robotics. 24(6): 1262-1273.

F. Yan, Y. Y. Wang, W. Xu, et al. 2018. Time delay control of cable-driven manipulators with artificial bee colony 
algorithm. Transactions of the Canadian Society for Mechanical Engineering. 42(2): 177-186.

H. S. Yoon and B. J. Yi. 2009. A 4-DOF flexible continuum robot using a spring backbone. 2009 International Conference on Mechatronics and Automation, IEEE.

H. Yamashita, K. Matsumiya, K. Masamune, et al. 2006. Two-DOFs bending forceps manipulator of 3.5-mm diameter for intrauterine fetus surgery: feasibility evaluation. International Journal of Computer Assisted Radiology and Surgery. 1: 218 .

I. D. Walker. 2013. Continuous backbone "continuum" robot manipulators. ISRN Robotics.

J. W. Suh, K. Y. Kim, J. W. Jeong, et al. 2015. Design considerations for a hyper-redundant pulleyless rolling joint with elastic fixtures. IEEE/ASME Transactions on Mechatronics. 20(6): 2841-2852.

L. Huang, L. Y. Yin, B. Liu, et al. 2021. Design and error evaluation of planar 2DOF remote center of motion mechanisms with cable transmissions. Journal of Mechanical Design. 143(1), 013301-1-12.

M. C. Lei and D. Ruxu. 2011. Geometry modeling and simulation of the tendon-driven bending section of a flexible ureteroscope. Proc. of the World Congress on Eng. and Comp. Sci. 2

N. Simaan. 2005. Snake-arm units using flexible backbones and actuation redundancy for enhanced miniaturization. Proceedings of the 2005 IEEE International Conference on Robotics and Automation, IEEE.

R. Buckingham. 2002. Snake arm robots. Industrial Robot: An International Journal. 29(3): 242-245.

T. Kato, I. Okumura, H. Kose, et al. 2014. Extended kinematic mapping of tendon-driven continuum robot for neuro endoscopy. 2014 IEEE/RSJ International Conference on Intelligent Robots and Systems, IEEE.

X. Dong, M. Raffles, S. C. Guzman, et al. 2014. Design and analysis of a family of snake arm robots connected by compliant joints. Mechanism and Machine Theory. 77: 73-91.

X. Dong, M. Raffles, S. Cobos-Guzman, et al. 2016. A novel continuum robot using twin-pivot compliant joints: design, modeling, and validation. Journal of Mechanisms and Robotics. 8(2): 021010.

Y. J. Kim, S. B. Cheng, S. Kim, et al. 2014. A stiffness-adjustable hyper-redundant manipulator using a variable neutral-line mechanism for minimally invasive surgery. IEEE Transactions on Robotics. 30(2): 382-395.

Z. Li and R. Du. 2013. Design and analysis of a bio-inspired tendon-driven multi-section flexible robot. International Journal of Advanced Robotic Systems. 10(4): 209.

\section{The list of figure captions:}

1. Fig. 1. A conventional tendon-driven snake-arm robot: (a) the straight configuration; (b) the bending state without external forces; (c) the S-shape state under the lateral force.

2. Fig. 2. Two contacting spherical magnets: (a) the initial state without lateral forces; (b) the state with lateral force.

3. Fig. 3. The robot design: (a) the CAD model of the snake-arm robot; (b) the disk of the manipulator; (c) the (c) The Author(s) or their Institution(s) 
initial configuration of the manipulator.

4. Fig. 4. Kinematics mapping of the snake-arm robot.

5. Fig. 5. The structure of the single rolling joint.

6. Fig. 6. The bending structure analysis in the single rolling joint: (a) the top view of the bending configuration; (b) the side view of the bending configuration.

7. Fig. 7. The maximum bending angle of the single rolling joint.

8. Fig. 8. The D-H coordinate system.

9. Fig. 9. The workspace of the manipulator: (a) the workspace with $\theta \in\left[0,20^{\circ}\right]$; (b) the configuration on the $\mathrm{x}-\mathrm{z}$ plane with different bending angles.

10. Fig. 10. The passive compliance analysis of the snake-arm robot.

11. Fig. 11. The prototype of the snake-arm robot.

12. Fig. 12. Load experiments under straight configuration: (a) unloaded straight configuration; (b) configuration under $4 \mathrm{~N}$ weight; (c) configuration under $5 \mathrm{~N}$ weight.

13. Fig. 13. Load experiments in bending configuration. 


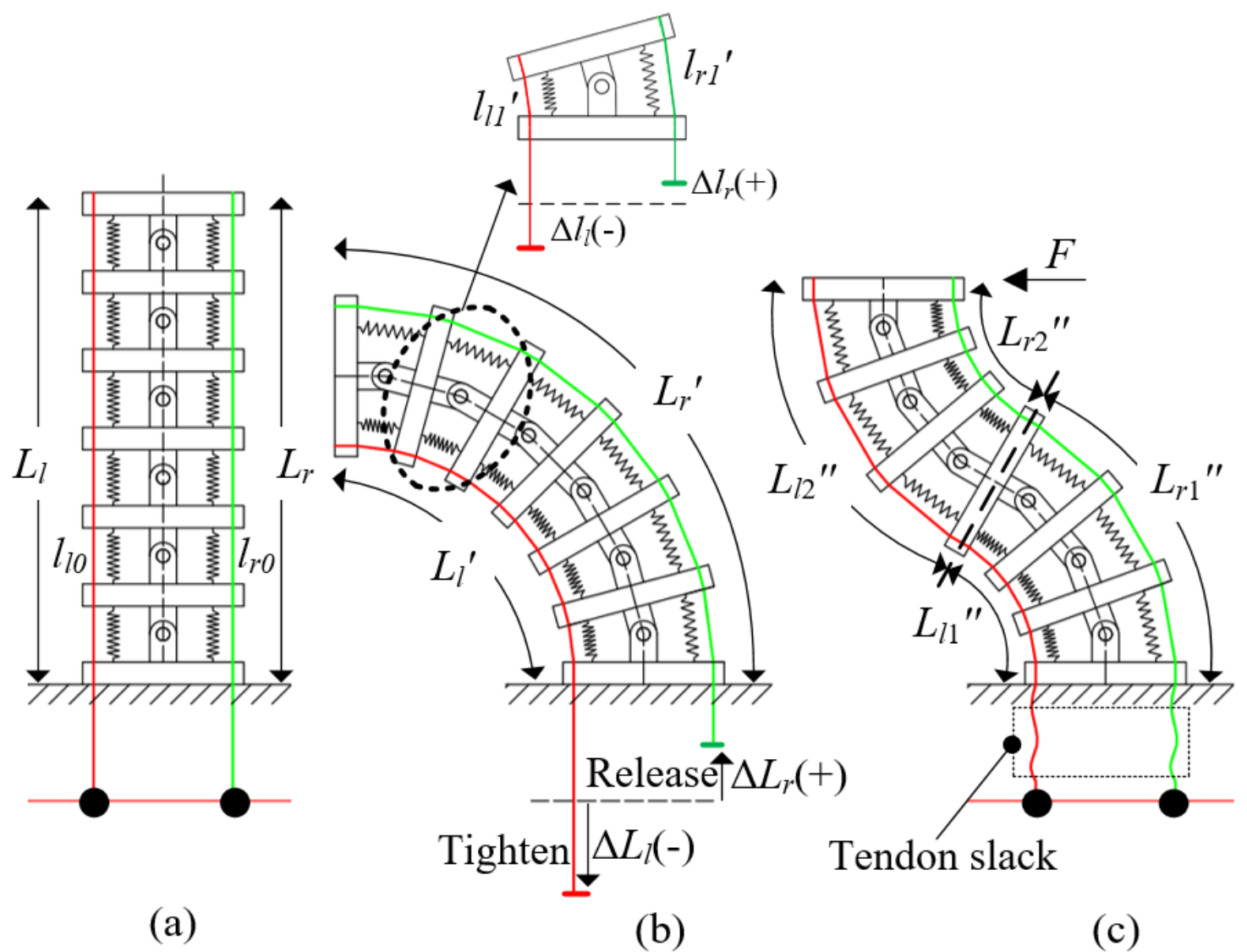

Fig. 1. A conventional tendon-driven snake-arm robot: (a) the straight configuration; (b) the bending state without external forces; (c) the $S$-shape state under the lateral force. 
(a)

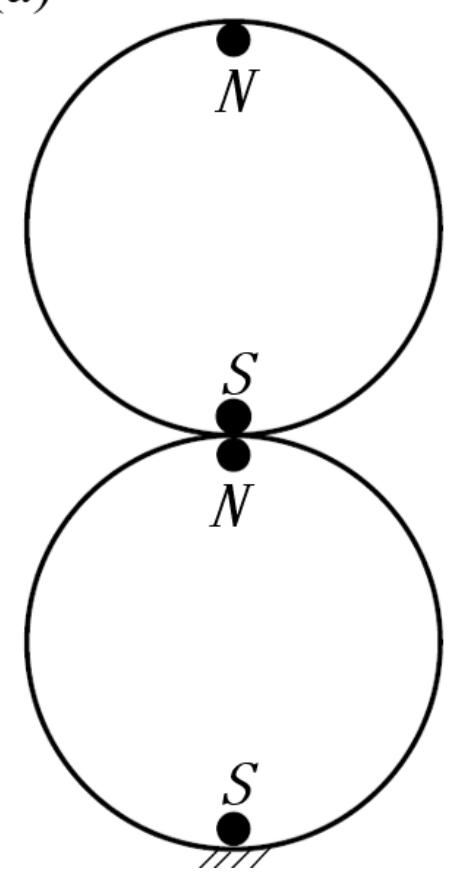

(b)

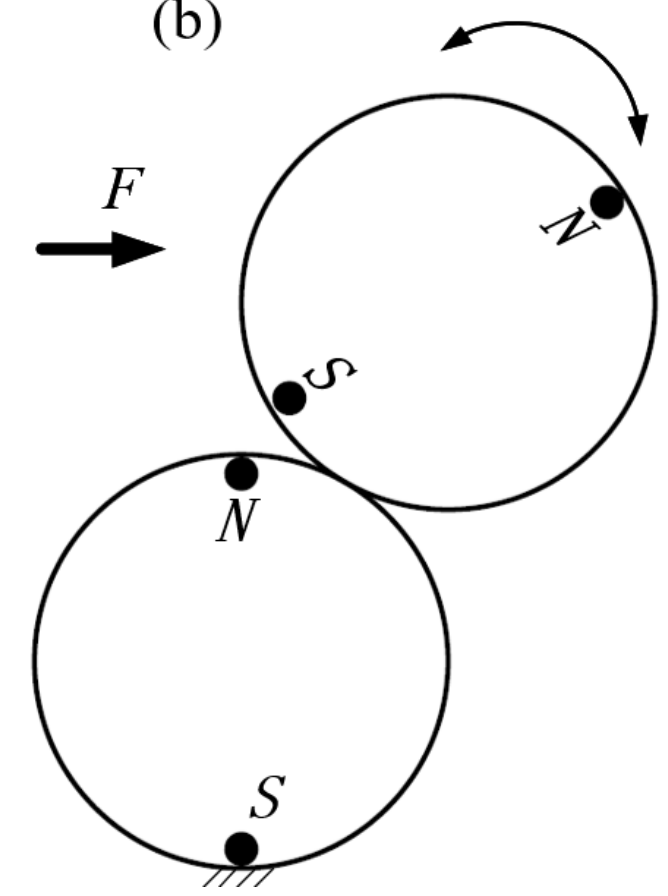

Fig. 2. Two contacting spherical magnets: (a) the initial state without lateral forces; (b) the state with lateral force. 


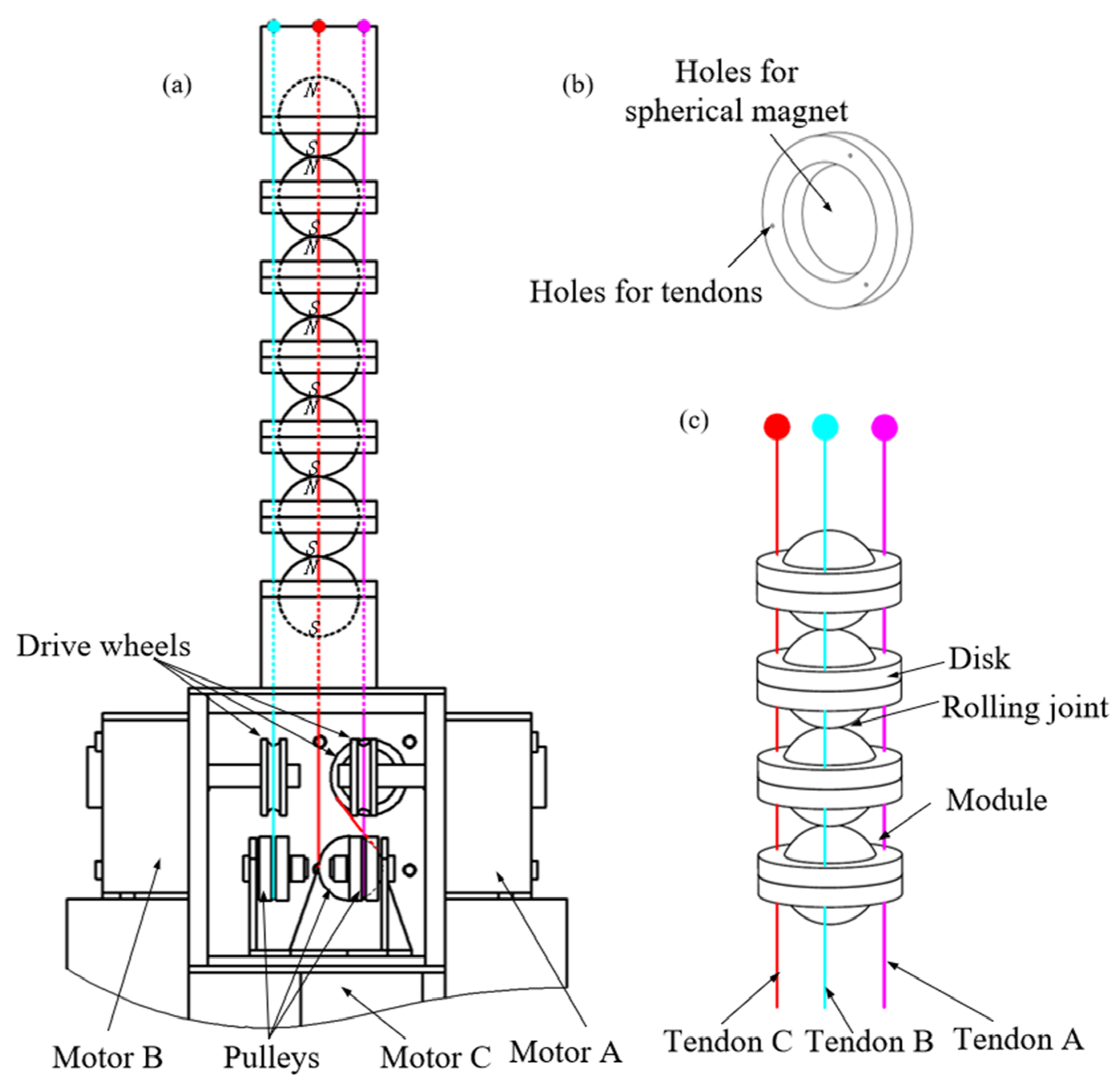

Fig. 3. The robot design: (a) the CAD model of the snake-arm robot; (b) the disk of the manipulator; (c) the initial configuration of the manipulator. 


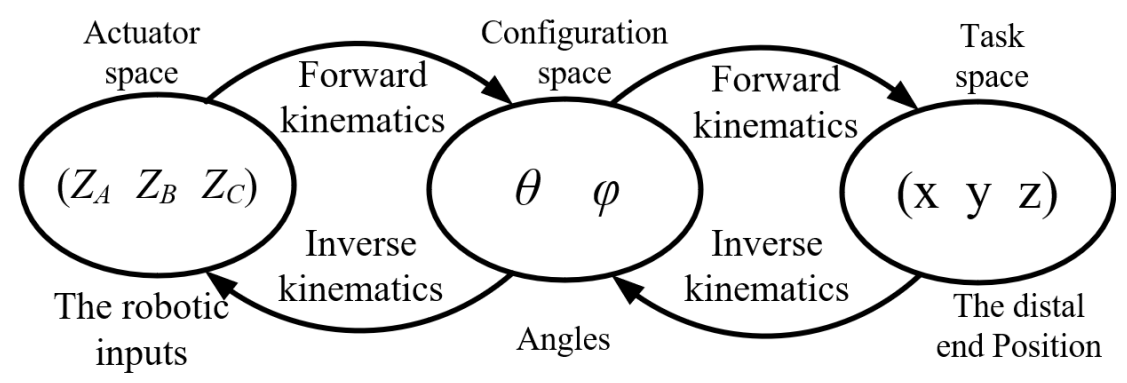

Fig. 4. Kinematics mapping of the snake-arm robot. 


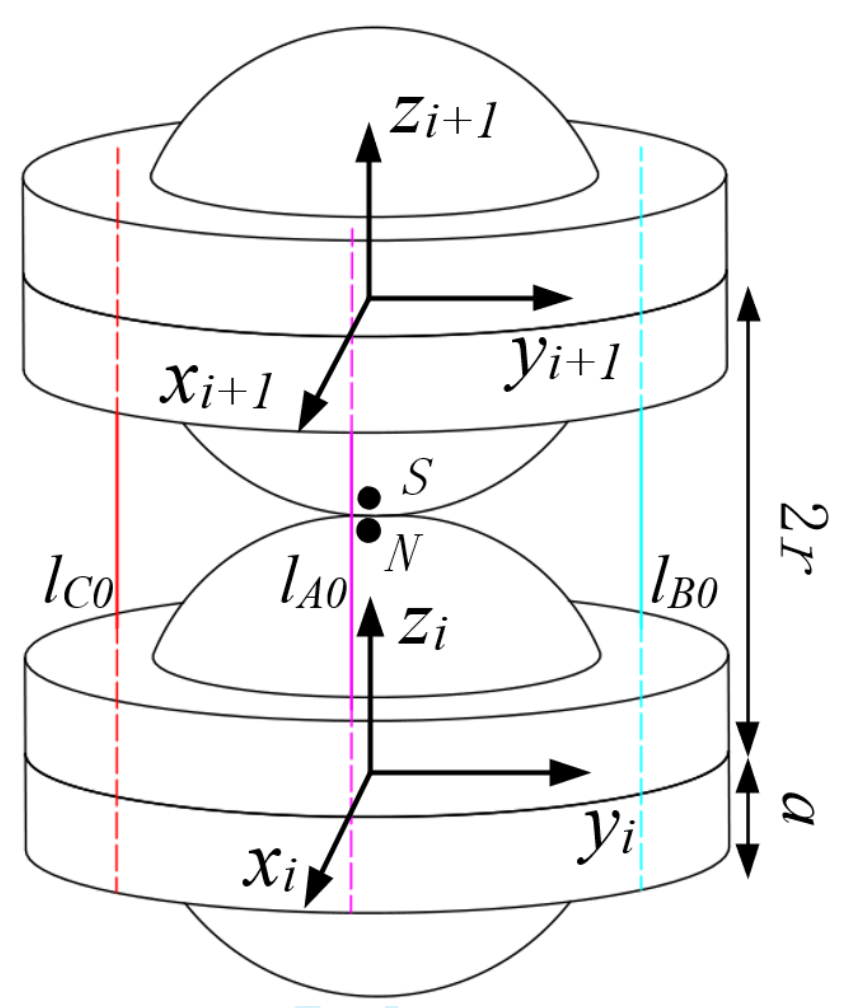

Fig. 5. The structure of the single rolling joint. 
(a)

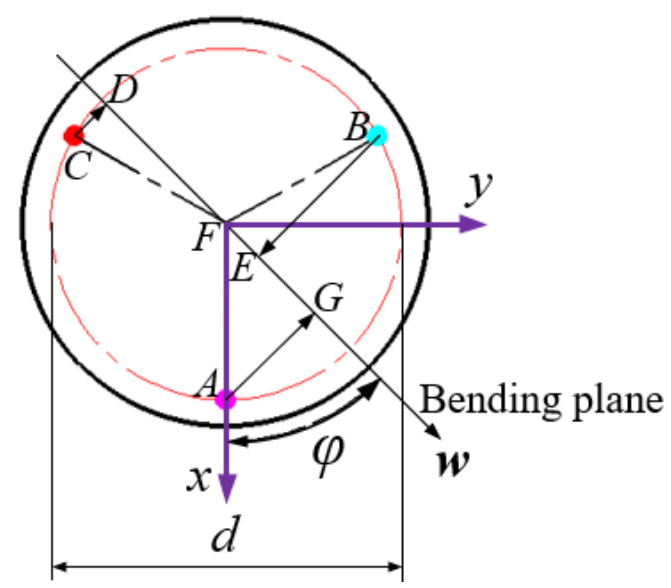

(b)

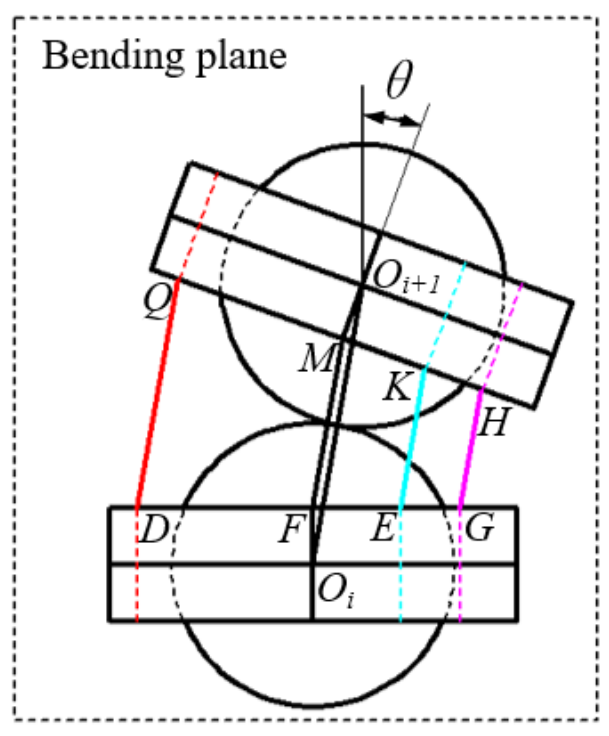

Fig. 6. The bending structure analysis in the single rolling joint: (a) the top view of the bending configuration; (b) the side view of the bending configuration. 


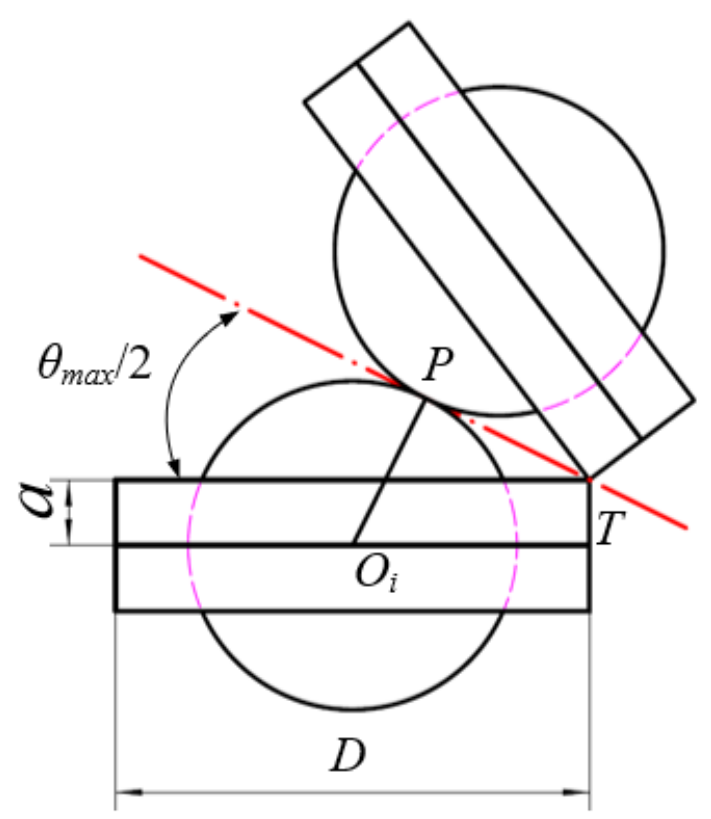

Fig. 7. The maximum bending angle of the single rolling joint. 


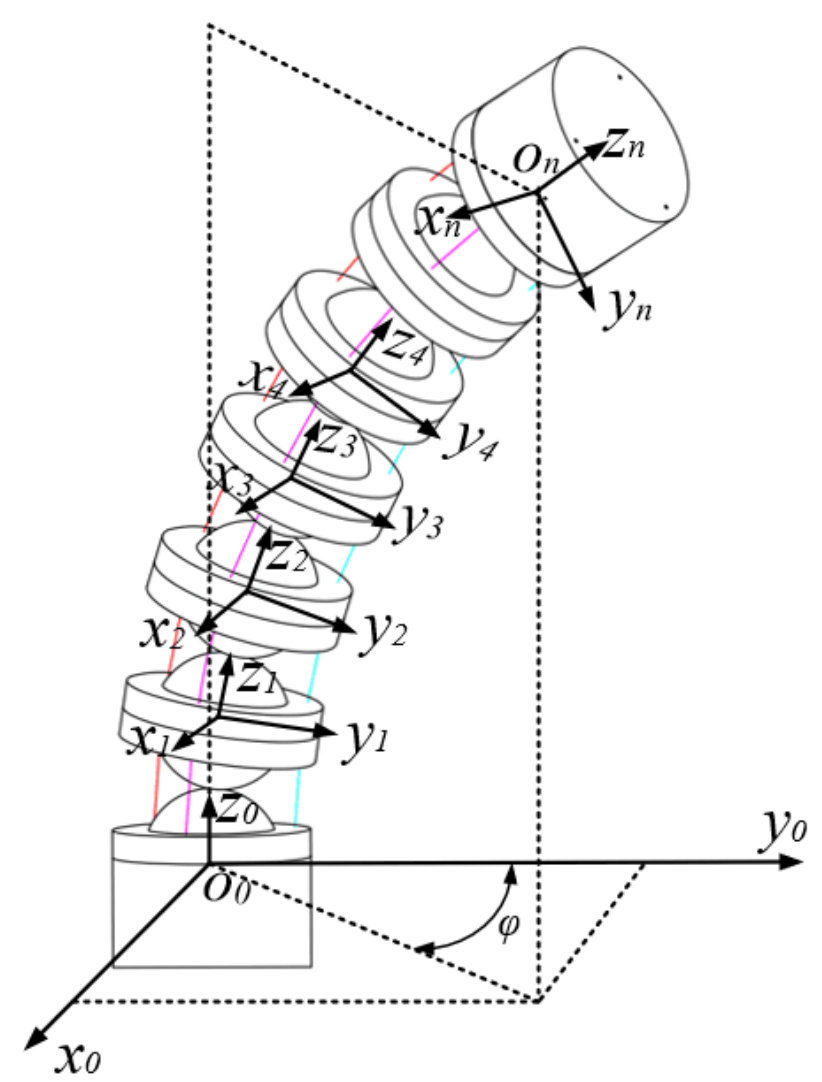

Fig. 8 . The D-H coordinate system. 
(a) $\theta \in\left[0,20^{\circ}\right]$

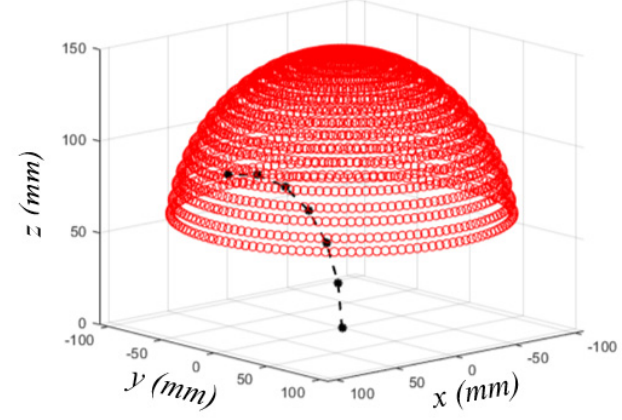

(b) The configuration on the $x-z$ plane

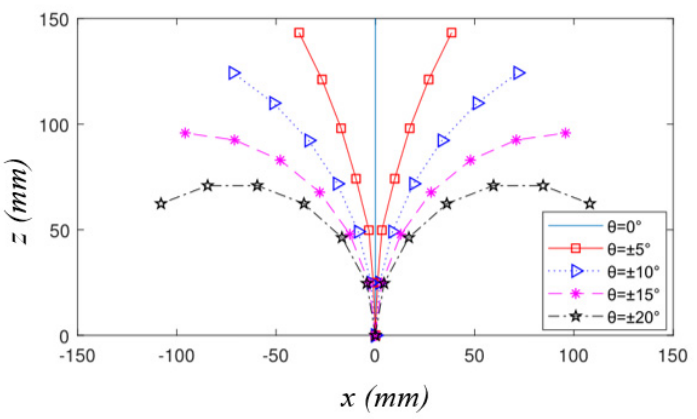

Fig. 9. The workspace of the manipulator: (a) the workspace with $\theta \in\left[0,20^{\circ}\right]$; (b) the configuration on the $x-z$ plane with different bending angles. 


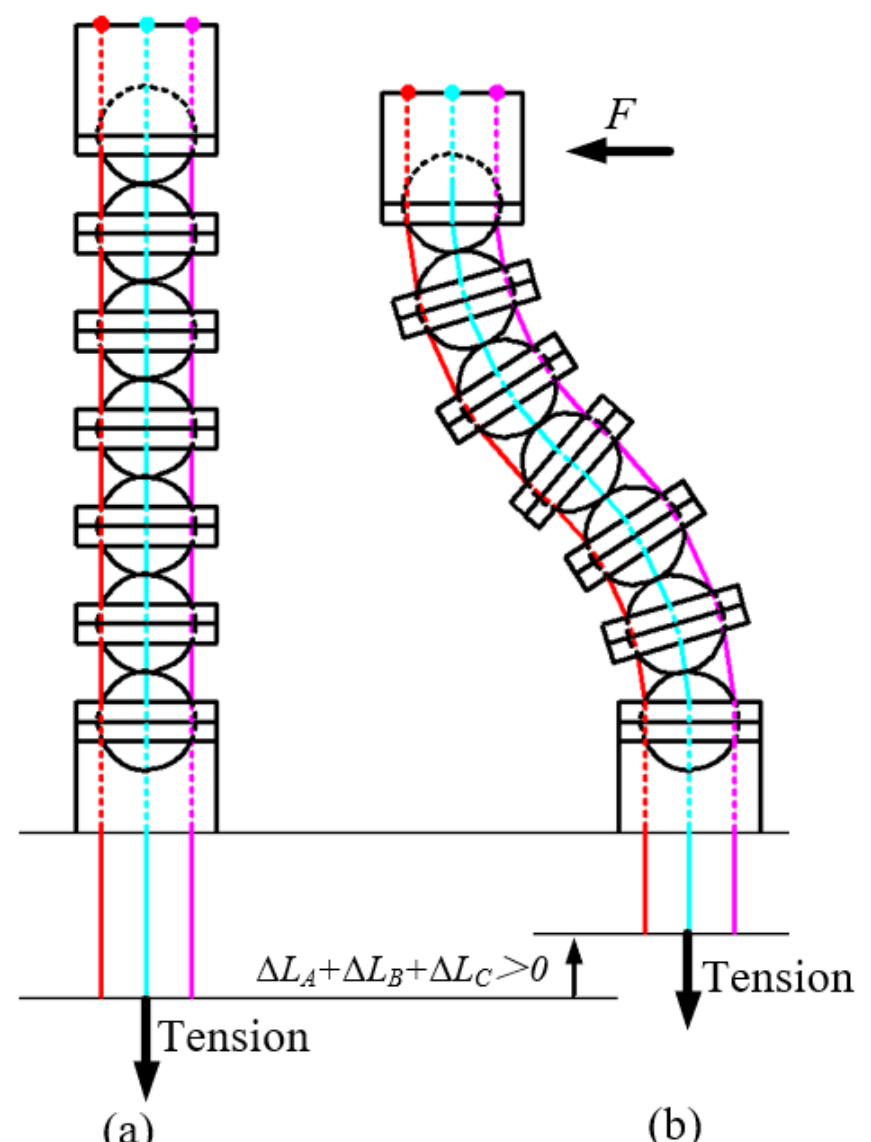

Fig. 10. The passive compliance analysis of the snake-arm robot. 


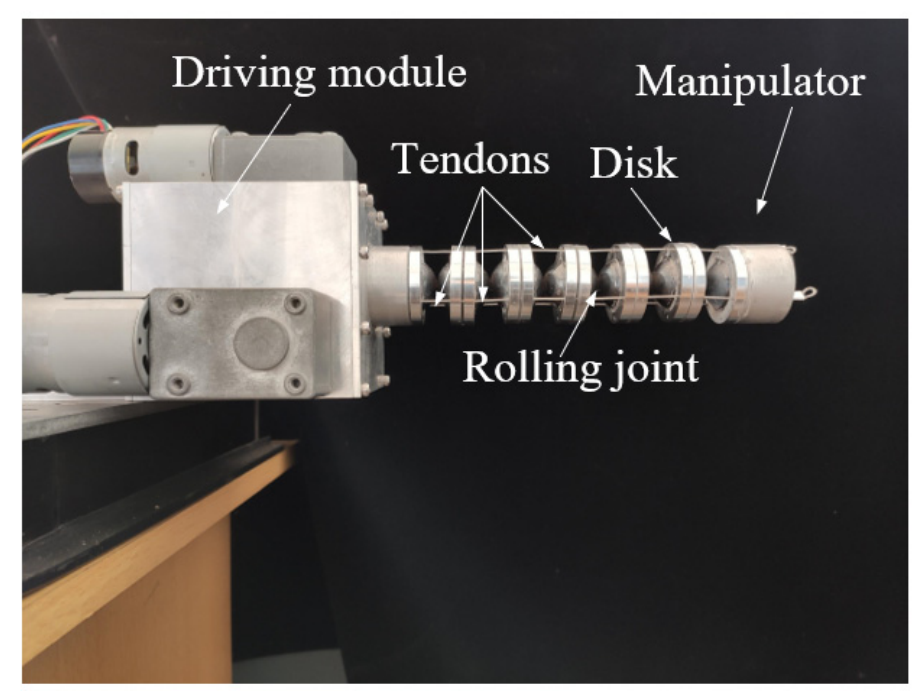

Fig. 11. The prototype of the snake-arm robot. 

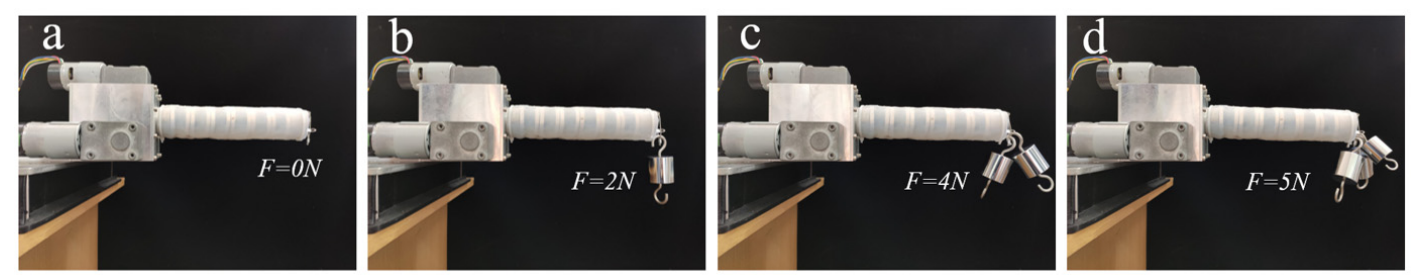

Fig. 12. Load experiments under straight configuration: (a) unloaded straight configuration; (b) configuration under $4 \mathrm{~N}$ weight; (c) configuration under $5 \mathrm{~N}$ weight. 

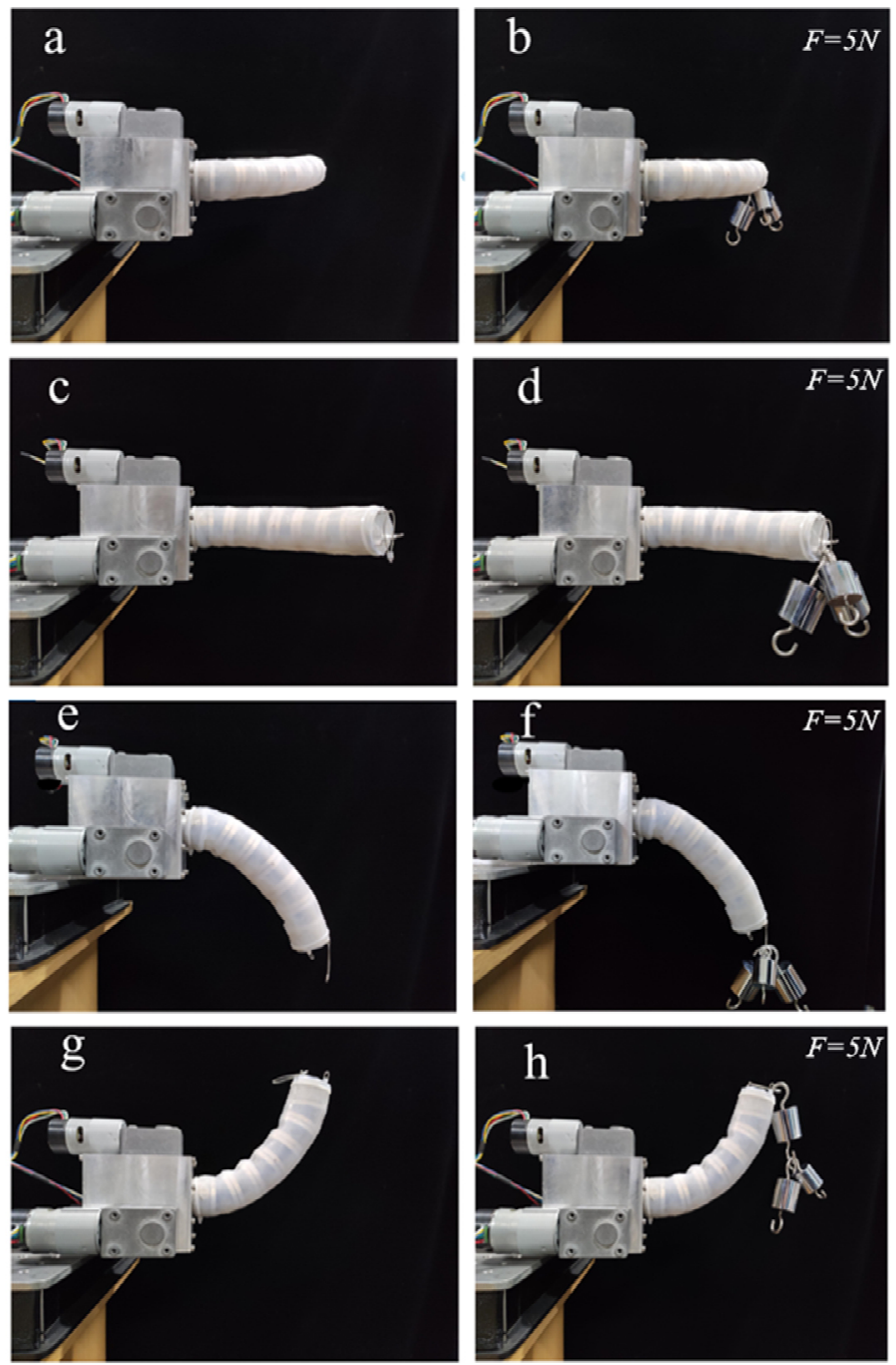

Fig. 13. Load experiments in bending configuration 